\title{
Using presence to evaluate an augmented reality location aware game
}

\author{
Rod McCall • Richard Wetzel · Johannes Löschner • \\ Anne-Kathrin Braun
}

Received: 17 June 2009/Accepted: 15 December 2009/Published online: 30 June 2010

(C) Springer-Verlag London Limited 2010

\begin{abstract}
Location-aware augmented reality games provide players with a rich and potentially unlimited range of interaction possibilities. In this paper, a study is described which uses a number of measurement techniques including questionnaires, direct observation, semi-structured interviews and video analysis to measure player's sense of presence. The paper points to the importance of the availability of actions within augmented reality games and how this shapes their sense of presence. The findings indicate that such an approach to measuring presence can provide valuable information on the structure of augmented reality location-aware games.
\end{abstract}

\section{Introduction}

Location aware games which use augmented and mixed reality technologies have the primary objective of in some way altering the players' perceived sense of reality, for example altering where they feel present socially, physically or temporally. This paper discusses the evaluation of one such game which takes place in a large European city, Cologne, Germany [10]. Playing a game with augmented elements in a city makes testing user experiences problematic due to the random uncontrollable nature of street life. Furthermore, testing concepts such as presence are even more problematic as to date most tests have taken place under very heavily controlled laboratory conditions. In order to achieve cohesion, the paper focuses on comparing player experiences at different points within the

R. McCall $(\bowtie) \cdot$ R. Wetzel · J. Löschner · A.-K. Braun Collaborative Virtual and Augmented Environments, Fraunhofer FIT, Schloss Birlinghoven, 53754 Sankt Augustin, Germany e-mail: rod.mccall@ fit.fraunhofer.de game using a range of techniques such as questionnaires, interviews and video analysis.

The paper initially presents some background literature which explores the nature of place and presence, information regarding the study methods, data obtained from the study and finally discussion and conclusion.

\section{Background}

\subsection{TimeWarp}

TimeWarp is an outdoor Mixed Reality game that allows for exploring the history of a city using AR and mobile devices. The background of TimeWarp is the tale of the elves of Cologne (as seen in Fig. 1). In this tale, some small elves which helped the residents of Cologne with daily chores suddenly disappeared. According to legend, they disappeared when they were discovered by a tailor's wife. In the game, we extend this legend by spreading the rumour that the elves actually never left Cologne, but instead fell into some time portals. As a result, they still within the city but are trapped within these time portals. The goal of the game for each player is to go to each time period (epoch) and rescue the elves. In order to help them in their task, the players are equipped with a "magic technical" system (see Fig. 2), which enables them to see the elves and to visit different time periods-roman, medieval, new age and even the future. In order to rescue the elves, the players must complete a series of challenges which relate to the City of Cologne and their current time period.

TimeWarp is a collaborative two-player game which uses ultra-mobile PCs (UMPCs) that are equipped with a variety of sensors capable of detecting the movement, 


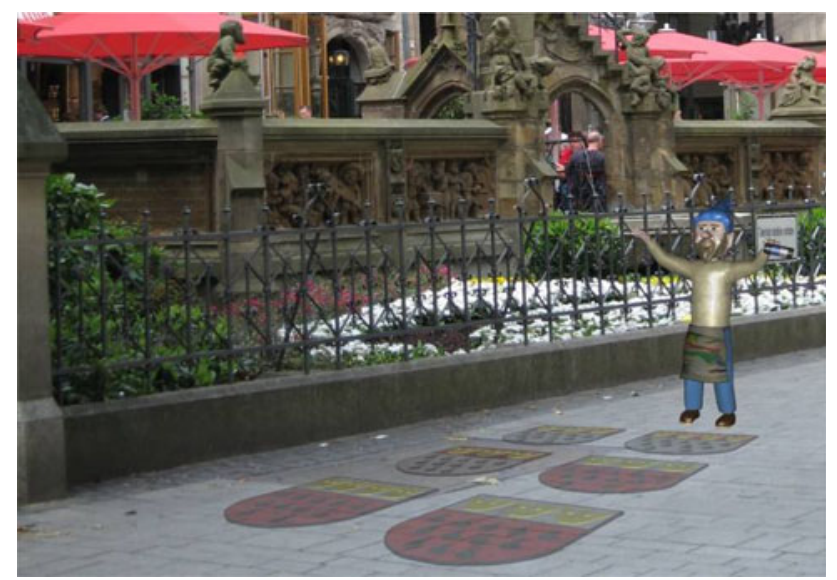

Fig. 1 An augmented character at one of the locations in TimeWarp

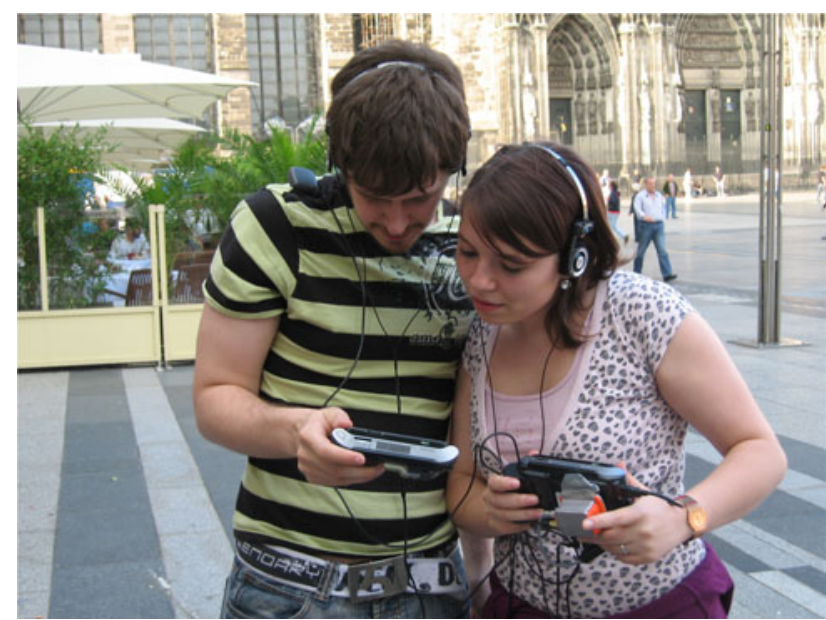

Fig. 2 Two players using an ultra-mobile PC for orienteering themselves

position and orientation of the players (via GPS and inertial sensors). Both players take on different roles in the game. One UMPC is used as a map and information device that enables the players to navigate through the city and find locations where the elves might be. The other UMPC provides a lens into the various time periods. The lens is effectively a video stream which receives input from a camera on the back of the UMPC, augmented elements such as characters, objects and buildings are then added to the scene.

Since TimeWarp's game area is open and cannot be limited by any physical boundaries, narrative structures are necessary to lead the players through the game experience. For that purpose, a number of virtual characters are used. The characters will talk to the players once they are within a certain range. The game contains 156 recorded voice segments recorded by various actors. To provide the game with more sophisticated and adequate acoustic feedback, a complete sound design developed. The process resulted in

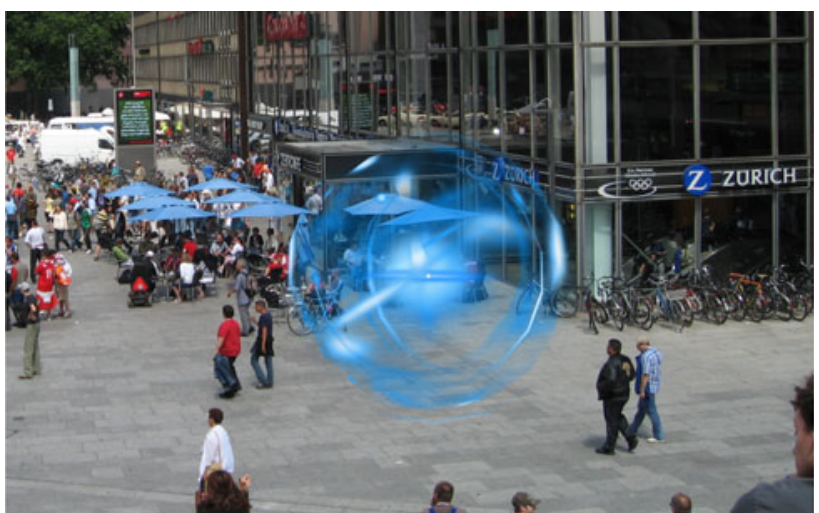

Fig. 3 A time portal in front of the train station

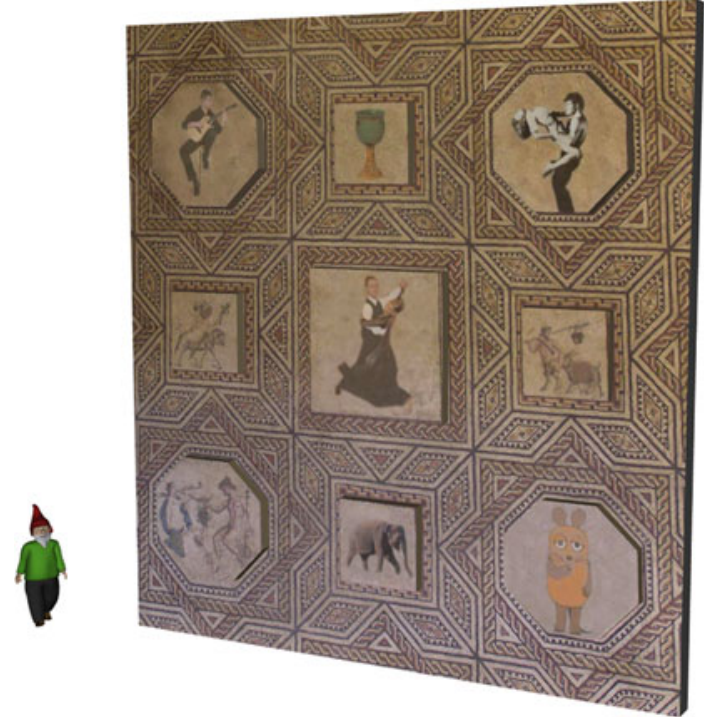

Fig. 4 An elf next to the Dionysus mosaic challenge

60 new sounds which have been added to the game in three different layers: interface sounds are played when a player interacts with GUI elements, object sounds are sounds that are spatially associated with virtual objects and a number of environmental sounds are scattered over the game area to support spatial presence in certain locations.

In order to travel to the different time periods, players can create time portals (see Fig. 3), they must then walk through these virtual objects in order to visit another time period.

Within each time period, players encounter an elf who must complete a challenge, as the elf is unable to complete this challenge they ask the players for assistance. One of the challenges in the Roman time is to help (re)create the Dionysus mosaic which today can be seen in the RomanGermanic museum (see Fig. 4). During the experience, many players spotted the original within the museum window and were able to use this as a guide when 
completing the virtual version. An example from the future time period is a space port where the players have to repair some of the orientation lights in order to assure the safe landing of an approaching spacecraft. In order to solve the challenges, the players typically have to point at the virtual objects and characters using their UMPCs and then click a button to start the interaction. Each interaction depends on the object or character at that location. In one example, the interaction involves rotating a piece of the mosaic, in another the user is able to turn on the spaceship landing lights, thus helping it to land safely.

\subsection{Related games}

One of the first augmented reality games was ARQuake which is a version of the famous first person shooter Quake [16]. In ARQuake, the player has to shoot monsters with the help of a gun-shaped interaction device while wearing a head-mounted display and a backpack containing a laptop. The player can move freely around in the environment and encounters various creatures that hide between real world buildings. Human Pacman [5] uses the same basic hardware setup (backpack with laptop and head-worn display) while converting the arcade classic Pacman into the real world environment of a university campus. The Pacman player has to walk around and collect virtual pills while being hunted down by the ghost players.

Epidemic Menace [12] is a crossmedia game applying augmented reality and other technologies to create a game in which players must clean a contaminated campus of lethal viruses while at the same time solving a detective story uncovering the identity of the villain behind all of it. Another mixed reality game with strong story-driven aspects is Interference [20]. Players become telecom engineers who have to uncover the mystery of a complete shutdown of all communication technology inside the city limits. Players are equipped with mobile phones and Ultramobile PCs which are used to contact non-player characters, interact with video snippets representing memories. They are also able to see a virtual version of the broken down network and the mythical world underneath it.

Although not an augmented reality game REXplorer [2] is a relevant location-based game as it also takes place within a city. However, it is targeted specifically at tourists visiting the city of Regensburg. The game makes use of a real gravestone situated in the city's church, and players interact with paranormal characters appearing on their smartphones using "magical" gestures.

Frequency1550 [11] is a game set in Amsterdam with aims to teach pupils about the medieval period of the city. Players are equipped with mobile phones and encounter characters from the past via small video clips while following the narrative and at the same time competing with

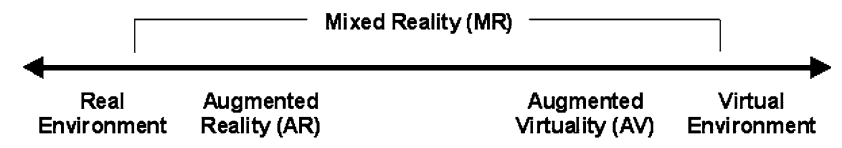

Fig. 5 Milgram and Kishno's mixed reality continuum

other teams. Visby [7] takes a similar approach and connects the players to the mythical side of the Swedish city Visby through the use of GPS location data, smartphones combined with audio feedback by characters from long lost forgotten times.

\subsection{Presence, place and flow}

The work undertaken within this paper was part of the EC funded IPCity project, which explores presence and interaction within city-based mixed reality environments. Although each example system within the project focuses on different elements, one overriding objective of the project is to understand the user experience, and as part of this feelings of presence and place within mixed and augmented reality spaces. In this context, presence is defined as the feeling of being within a particular place or with another (real/virtual) person.

There are a number of definitions of sense of place and as such a commonly agreed upon model does not exist. However, two broad approaches seem relevant to the work here, for example Relph [17] indicates that sense of place is derived from: physical properties, activities and meanings [17] where as a sociological perspective would say that it is derived from the relationship between the player (self), environment and others [9]. While both of these definitions apply to physical environments they are also applicable to games such as TimeWarp. For example players move around with a new hybrid space consisting of real and virtual elements, this it could be argued constitutes the physical dimension of Relph's definition. The augmentations in turn shape the actions available and the meaning which are drawn from various locations. Similar parallels can also be drawn within the Gutavson model, where the environment is the hybrid world and others can be seen to constitute non-participants and other players. However, regardless of the model chosen, the design of the game is essentially intending to alter the place in which the game takes place.

Milgram and Kishino's [14] Mixed Reality Continuum (see Fig. 5) provides a useful starting point to explore the concepts of presence and place. It indicates that experiences exist within range from purely real to purely virtual with augmented reality and augmented virtuality acting as middle points. TimeWarp is an augmented reality game and therefore occupies the left side of the continuum. However, as the experience consists of real and virtual 
elements, and as some game aspects force interaction with purely virtual aspects it becomes important to explore how the various realities are blended, where people feel present e.g. more in the virtual or real sphere and finally what gives rise to any feelings of changes in presence. This has an impact on the precise definition of presence within such experiences and the measurement techniques adopted later.

Although the MR continuum is perhaps a simplification of such experiences, it does point to the fact that there is no clear division between the different realities. Indeed, it indicates that such experiences are by default a blend of the meditating technology and real elements. This would appear to point to the view taken by Gibson [8] that there is no fundamental difference between real or virtual experiences and that both are mediated. Gibson further extends this by indicating that the origin of our perception within such environments is filtered through our actions and purposes rather than the environment itself. Furthermore as Mantovani and Riva [13] argue, presence is also derived from social and cultural dimensions, as actions and the need for actions are always motivated by social elements and as a result reality is co-constructed. These aspects are of particular relevance within augmented reality games where the player is driven by the need to successfully complete specific actions in order to finish the game. Thus, the core factor in their perception of these new realities is how the gaming elements bring together the real and virtual aspects through the availability of actions. The available actions will subsequently shape the ability of the players to complete their tasks. Therefore, presence in the context here is not deemed to be the idea of sensory substitution such that people are immersed in a new experience, as is the case with virtual environments. Rather in the context of location-based mixed reality games, the sense of presence of where people feel within the game is shaped by their actions the game provides. This approach to presence has many commonalities to the idea of game flow [18], which is drawn heavily from the work of Csikszentmihalyi [6]. Immersion from the perspective of flow is related more to the ability to easily complete tasks such that within a gaming context the players lose track of self and time and the involvement becomes effortless.

The blurring of boundaries also extends to the game space, or magic circle within location-aware games. Montola [15] for example points out the different forms of ways in which the magic circle can be expanded through social, temporal and spatial means. At the most basic level, the magic circle which a game occupies as opposed to the reality in which it is situated e.g. the players (social), rules of engagement, objects or actions, specific area where a game takes place (spatial) in contrast to the aspects of reality such as the room in which the game is player, or the period of time in which it is played (temporal). All three of these elements (spatial, temporal and social) may become expanded within pervasive games.

In traditional games such as Monopoly, the magic circle is quite clear for example while many people may be within a room only a certain number of them will be the players who undertake the actions of buying and selling property; these actions are said to exist within the magic circle as they relate only to the game. Furthermore, the additional aspects such as the room furniture do not play a part in the game itself. However, within pervasive and augmented reality games such as TimeWarp, the border of the magic circle becomes less clear as non-players can have an effect on the game, e.g. stopping the players to ask directions or to ask questions. This in turn results in social expansion as those who are part of the game extends beyond the pre-selected players and may include those who are unaware they are part of the gaming experience. However, it should be noted that in TimeWarp while players can interact with passers-by it is not a specific element of the game design as is found in Uncle Roy is All Around You [3].

The spatial dimension also increases as not only do players interact with the game content but also the wider environment for example by navigating between real locations, it can also extend to how people behave and interact with real world elements even if these were not included in the game design. Such expansions have an important impact on where the player and with whom they will feel present as unlike the strict laboratory experiments which are often used for testing virtual environments it becomes impossible to completely remove uncontrollable variables from the experience. However, this is a key aspect of such experiences and thus should form the basis of the measurement approaches and underlying topics for exploration.

\subsection{Methods}

TimeWarp made use of questionnaires, in combination with interviews, direct observation and video analysis. We started by exploring existing Presence questionnaires. However, these were not always suited to the evaluation settings or the types of experience being explored. Furthermore, such questionnaires had to support assessment of physical Presence (including where the user felt location in the Mixed Reality experience), social Presence (with real and virtual people) and sense of place. Additionally, we also had to explore if the users felt present in different time periods (temporal Presence). For this task, we chose to build upon the MEC spatial Presence questionnaire [19] by adding questions specifically related to the TimeWarp experience. The primary changes to MEC included adding questions which specifically explored the blending of 
experience and the comparison between real and virtual elements, including non-game participants and altering the scale used to collect data. MEC itself was insufficient for exploring issues to do with social presence, in particular with respect to virtual characters. It was for this reason that we added questions from the Bailenson et al. [1] social Presence questionnaire. We also added some questions from the Place Probe [4] to find out about which place(s) people felt they had visited as they took part in the experience; these were also modified to reflect aspects of TimeWarp, in particular the temporal dimension. However, questionnaire-based approaches only provide small hints as to the overall experience that the user has within such environments. In particular, they are not suitable for identifying where breaks or changes in presence occur. While the majority of users were video-taped, some were also observed as they took part in the game. For this, we adapted an observation technique developed within the EU project IPerG (http://www.iperg.eu), and used it to consider which notes were taken and also to act as a method of analysis for the videos. As players take part in the game their interactions are logged as follows: player-player interaction, player-device interaction, player-spectator interaction and player-game interaction management. The IPerG method proved useful while observing people although not all aspects were relevant. Because of that the method was slightly altered for the video analysis. For each interaction, it was noted into which categories the interaction could fit and which of the players was taking part. That allowed us to code more complex interactions, e.g. when both Players run towards a time portal while talking with each other. Additionally, the game-management category was removed as it has no relevance for TimeWarp.

A semi-structured interview approach was used to capture additional information based on preliminary observations for example when players entered or left a time portal. Questions were also based on questionnaire responses, in particular where feelings were particularly strong or appeared to contradict one another. Photographs of key elements of the game e.g. the time portals were also used to stimulate discussion.

We approached the data analysis from an independent perspective, for example each source was analysed independently; however, on completion we looked for commonalities in the results. Additionally, we used observation notes from the participants to derive questions during the interview sessions. This approach allowed the evaluators to drill down to discover specific items of interest.

\subsection{Participants and duration}

A total of 12 test runs were performed with 24 participants aged between 22 and 40 years. Eighteen of them were men and six women. Sixteen of the 24 were IT professionals or students in computer science. Four of the 16 were familiar with Augmented Reality. Broadly speaking, the only limiting factor in each game was the battery life of the UMPCs which typically restricted any gaming session to at most $90 \mathrm{~min}$. The questionnaire and interview sessions were conducted immediately after the experience and could last for up to $1 \mathrm{~h}$.

\section{Results}

\subsection{Questionnaire data}

For brevity the following results do not report all aspects of the questionnaires but instead focus on descriptive statistics relating mainly to feelings of presence and in particular where these change with respect to virtual and real elements or when players experience different time periods. The remainder of the data within the questionnaire focuses on background information such as cognitive aspects, visual spatial imagery and usability. As with many of these games technical challenges often cause problems for end users, therefore where appropriate basic usability issues relating to these aspects are not reported. It is acknowledged that technical and usability factors do have an impact on game play and will impact on sense of presence.

In the statistics presented later in this paper, we have chosen to focus primarily on the means accompanied by standard deviations. The mode and median scores are then presented in order to provide an additional level of detail, for example to indicate if when aspects such as outliers are removed the same outcome remains.

The first part of the questionnaire provided data relating to attention, in particular whether this was focused more on real or virtual aspects. In this section, the players were asked to rate each question on a scale of $1-7$, with a score of 1 indicating a tendency towards the real elements, a score of 7 indicating more towards the virtual elements and a score of 4 no opinion. The remaining part of the questionnaire was scored using a rating scale where a score of 1 for strongly agree and 7 for strong disagree, with 4 being no opinion.

During the test, we were interested in uncovering the differences which people felt towards the real and virtual elements whether this related to people, characters, objects or locations. It was found for example that players perceived game actions as taking place within the virtual space $(\mathrm{Q} 3$ : mean $=6.28$, stdev $=1.13$, mode $=7$, median $=7$ ) and that this subsequently resulted in them focusing their attention on the virtual world (Q1: $\mathrm{m}=4.72$, stdev $=1.23$, median $=5$, mode $=6$ ) and concentrating on the game elements more strongly than 
reality $(\mathrm{Q} 2:$ mean $=5.28$, stdev $=1.32$, median $=5.5$, mode $=6$ ). This may have been caused by the fact that the players felt the game elements did not feel real (Q11: mean $=5.28$, stdev $=132$, median $=5.5$, mode $=6$ ). Interestingly though they noted that they navigated using the real environment $(\mathrm{Q} 7:$ mean $=3.06$, stdev $=2.21$, median $=2.5$, mode $=1$ ) and felt more part of the real than game space $(\mathrm{Q} 5$ : mean $=3.75$, stdev $=2.24$, median $=3.5$, mode $=1)($ Table 1$)$.

Another key element of the study was the changing sense of presence which people feel either as they visit different time periods while visiting different locations or when they enter or leave the game. Players indicated they did not feel any change in temporal presence as they visited the various locations. However, they indicated that they felt present within a game $(\mathrm{Q} 13$ : mean $=3.17$, stdev $=1.82$, median $=3$, mode $=2$ ), this fact was further emphasized by the fact that they felt a strong change in location on leaving the game experienced (Q16: mean $=5.33$, stdev $=1.57$, median $=5$, mode $=5$ ) as opposed to when entering it $(\mathrm{Q} 14$ : mean $=3.61$, stdev $=1.71$, median $=4$, mode $=2$ ). Other responses within the questionnaire pointed to some preferences in locations with the future time period being the most well liked (eight responses) a view shared when asked about what was their favourite location (eight responses). The roman time period was the second most popular with four responses (Table 2).

As noted earlier, it is the availability of actions which has a significant impact on the players' sense of presence. Players noted that they felt a difference when interacting between real and virtual aspects (Q19: mean $=4.89$, stdev $=1.18$, median $=5$, mode $=5$ ), and that they felt more able to interact with virtual objects (Q20: mean $=3.22$, stdev $=1.73$, median $=3$, mode $=3$ ) more than real objects $(\mathrm{Q} 21$ : mean $=4.24$, stdev $=2.05$, median $=4$, mode $=2$ ). This is not surprising as the game did not specifically support interaction with real objects. The players felt that some degree of interaction was possible with virtual characters $(\mathrm{Q} 22$ : mean $=4.50$, stdev $=1.98$, median $=5$, mode $=2$ ) but as noted later they did not feel that the characters were real.

Actions are not purely related to objects, indeed the feeling of presence towards the co-player, non-participants and virtual characters are also important. The co-player had by far the largest influence on user's behaviour when compared to the other people who formed part of the
Table 1 First set of questions. Answers reaching from $1=$ real to $7=$ virtual. Graphs showing mean value

\author{
Question \\ Q1: I devoted my whole attention on \\ the [real/virtual]
}

$1=$ real $; 4=$ no opinion; $7=$ virtual

Q2: I concentrated on the [real/virtual]

Q3: Game actions took place in the [real/virtual]

Q5: It felt like the objects surrounded me [real/virtual]

Q7: When I walk around I mainly navigated using objects in the [real/ virtual] environment mean $=5.28$, stdev $=1.32$, median $=5.5$, mode $=6$

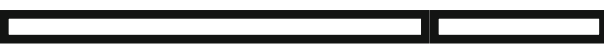

mean $=5.28$, stdev $=1.32$, median $=5.5$, mode $=6$

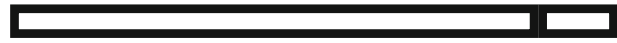

mean $=6.28$, stdev $=1.13$, mode $=7$, median $=7$ mean $=3.75$, stdev $=2.24$, median $=3.5$, mode $=1$

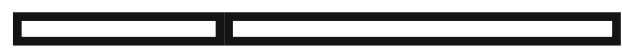

mean $=3.06$, stdev $=2.21$, median $=2.5$, mode $=1$ 
Table 2 Second set of questions. Answers reaching

from $1=$ strongly agree to

$7=$ strongly disagree. Graphs

showing mean value

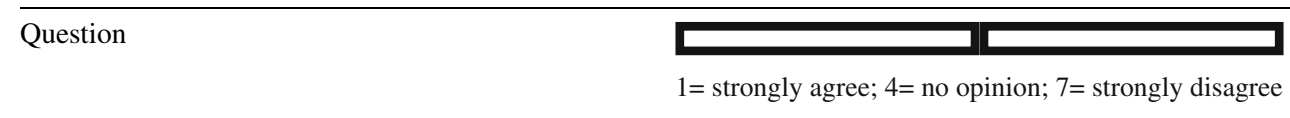

Q11: The virtual elements felt real

mean $=5.28$, stdev $=132$, median $=5.5$, mode $=6$

mean $=3.17$, stdev $=1.82$, median $=3$, mode $=2$

Q13: I felt I was present in a game which was not part of reality

Q14: I felt I was present in the overall experience and did not differentiate between real and virtual

mean $=3.61$, stdev $=1.71$, median $=4$, mode $=2$

mean $=5.33$, stdev $=1.57$, median $=5$, mode $=5$ when I left the game and returned to reality

Q19: I felt the same when interacting with real and virtual aspects

mean $=4.89, \operatorname{stdev}=1.18$, median $=5$, mode $=5$

mean $=3.22, \operatorname{stdev}=1.73$, median $=3$, mode $=3$

Q20: I felt I could interact with the virtual objects

Q21: I felt I could interact with the real objects

mean $=4.24$, stdev $=2.05$, median $=4$, mode $=2$

mean $=4.50$, stdev $=1.98$, median $=5$, mode $=2$ experience. It was also noted that the computer-generated characters were not perceived as real and that as noted earlier the ability to interact with virtual characters was not very strong.

The questionnaire also asked people to provide a set of words which best described their experience. Although many were related to technical issues, of the remaining responses the experience was rated interesting (nine times), fun or funny (nine times). They also commented on it being a new experience (four times), challenging (four times) and that it blended realities (three times). When asked about their views on the virtual elements, many commented that they felt misplaced (four times), other general comments included that it could benefit from more interactive elements (two times). The latter point also appeared within the responses to when players felt least present with two highlighting the walk between locations as a time when sense of presence dropped. 
Interestingly the same player said the future time period made them feel both most and least present. Sound in various forms (e.g. narrative or cues) made people feel most present (four times).

\subsection{Interview data}

The interviews were recorded and annotated by one of the evaluators. Based on the notes of the interviews they were then coded, and a record kept of the number of instances of the same response for each player. Only one incidence of an event per player is recorded, for example where the same or similar comment is made by the same player it counts as one incidence.

Rather than focusing on all the interviews, the data here explores the results relating to the time portals, the future time period, the mosaic challenge and co-operative elements. With respect to the time portals players found them fun (four responses) and the sounds (three responses) were also rated positively. They were also found to be interesting and exciting. The future time period was also well received with a number of positive comments including it being the players favourite (2), easy to solve (1), worked well (1), enjoyable (1) and with a nice task structure (1). Interview data from the Mosaic provided similar results with it being classed as a cool task (2) favourite (1), their favourite (2) and that it was clear what to do (1). The co-operative elements of the game were well received, with all players agreeing that this approach was effective. This approach was deemed to have made solving problems easier; however, some players (five responses) indicated they would like the second player to be incorporated more into the game and that this player may feel bored. The relative ease with which players could complete tasks was also commented on within the interviews, with comments such as confusing, hard to solve or easy being mentioned on various occasions across different challenges.

Negative interview comments were generally related to GPS aspects, for example floating objects which in turn made interacting or walking through elements such as Time Portals quite difficult (Table 3).

\subsection{Video analysis}

\subsubsection{Time portal interactions}

One typical interaction during the game involved the time portals that the players could create whenever they wanted to change into a different time period) (see Each time portal appears for $30 \mathrm{~s}$ and is $5 \mathrm{~m}$ away from the players Table 4). It should be noted however that the first time
Table 3 General overview of interaction types

\begin{tabular}{lrrr}
\hline & & $(\%)$ & Average per game \\
\hline Total amount of interactions & 476 & 100 & 60 \\
Player-player interactions & 250 & 53 & 31 \\
Player-environment interactions & 269 & 57 & 34 \\
Player-device interactions & 407 & 86 & 51 \\
Player-spectator interactions & 6 & 1 & 1 \\
Player 1 interactions & 450 & 95 & 56 \\
Player 2 interactions & 395 & 83 & 50 \\
\hline
\end{tabular}

Table 4 Observations concerning time portals

Total amount of time portals

Very sudden movement of players

Players running

14

Players laughing

8

portal is created automatically. Each time portal appears for $30 \mathrm{~s}$ and is $5 \mathrm{~m}$ away from the players.

During the games, 40 time portals were created. Typically players would be holding the UMPC high up when creating the time portal and then suddenly start moving together towards the time portal (moving together was necessary as the players' headphones were connected to the same UMPC). In 14 of these 40 occurrences, players would not only move swiftly but rather run towards the time portals in order to make sure to catch them. Eight times players were visibly laughing while trying to catch the time portal or directly afterwards. Five out of the eight seemed to be suffering from bad GPS data coming in thus catching the time portal became more difficult for the players as it would move away from them. While this was clearly frustrating for the players, it also within limits provided a certain degree of entertainment.

\subsubsection{Solving of challenges}

The game consisted of a total of five challenges: one tutorial challenge and one challenge for each time zone (see Each time portal appears for $30 \mathrm{~s}$ and is $5 \mathrm{~m}$ away from the players. Table 4). At each challenge, players were greeted by an elf explaining the dilemma and giving the players hints on what to do. Typical interactions included aiming at a virtual object and selecting it. Depending on the challenge this would mean picking up an object or manipulating it (e.g. rotating stones of a mosaic). Not all players managed to play all challenges (Table 5).

Players encountered a total of 37 challenges. It was noted that on 31 there were significant changes in movement, on 48 occasions players stood completely still for a significant period of time while they tried to solve 
Table 5 Observations when players were solving challenges

\begin{tabular}{lr}
\hline Total amount of challenges & 37 \\
Players changing positions & 31 \\
Players standing still & 48 \\
Players concentrated & 41 \\
Player 2 points at screen & 15 \\
Players laughing & 11 \\
Visible celebrations after & 9 \\
$\quad$ solving of challenge & \\
Signs of frustration & 12 \\
\hline
\end{tabular}

Table 6 Observations while players were walking around between challenges

\begin{tabular}{llll}
\hline & Total $\begin{array}{l}\text { On path to first } \\
\text { challenge }\end{array}$ & $\begin{array}{l}\text { Without path to } \\
\text { first challenge }\end{array}$ \\
\hline $\begin{array}{l}\text { Walking between } \\
\text { challenges }\end{array}$ & 31 & 8 & 23 \\
$\begin{array}{c}\text { Lots of device } \\
\text { checking }\end{array}$ & 16 & 7 & 9 \\
$\begin{array}{c}\text { Occasional device } \\
\text { checking }\end{array}$ & 4 & 0 & 4 \\
$\begin{array}{c}\text { No or almost none } \\
\text { device checking }\end{array}$ & 10 & 1 & 9 \\
\hline
\end{tabular}

challenges. On 41 occasions we observed the players concentrating very heavily on the challenge.

Due to the nature of the game, layer 2 could provide verbal advice or as was the case on 15 occasions point at the screen of the other player's UMPC. On 11 occasions the players were laughing during a challenge, while on nine occasions they celebrated the successful completion of a challenge with obvious gestures like giving thump's up or high-fiving. On 12 occasions players showed signs of frustration; this usually occurred when they had problems interacting with the virtual content.

\subsubsection{Between challenges}

The challenges themselves did not require the players to move around much, so most of walking around the city was done between challenges (see Table 6). Additionally, virtual content was only available at the challenges themselves.

While the players would refer to their devices regularly at the beginning of the game (especially on the way to the first challenge), this enthusiasm quickly disappeared and later on players would often only refer to devices to check if they had arrived at the correct location in order to complete a challenge.
Table 7 Observations concerning audio clues

\begin{tabular}{ll}
\hline Total amount of audio clues & 30
\end{tabular}

Players standing still while listening 20

\subsubsection{Audio clues}

After travelling through a portal, players were greeted by a guide giving them some background information about the newly reached time period (see Table 7). When players met with elves, these would also start talking to the players. These instances are not included in this table as it was impossible to tell from the video when players would actually get to talk with the elves. All audio information was provided through headphones worn by the players.

In total, we observed 30 occurrences of the players listening to audio, either at the game start, after having solved a challenge or when travelling to a new time zone. There were 20 instances of players standing completely still when listening to the information provided to them by the narrator. At all other times, the players would listen while walking.

\subsubsection{Player-spectator interactions}

As the game does not require the players to explicitly interact with non-players, there was been very few playerSpectator interactions (see Table 3). All of them were driven by spectators noticing that something was going on. Except in one instance where the players were stopped by a passer-by and asked what they were doing. On one occasions a passer-by threatened to hit the UMPC out of the players hand if they did not point it in another direction; however, because of being heavily involved in the game the player did not notice this.

\section{Discussion}

The game presented here was for the most part perceived by users as being part of a virtual world. This was reflected in the early questionnaire data which pointed to people attending more to the game environment, with the notable exception of when they navigated between locations. In the latter example, it was reported that they navigate using the real space and as noted in the observation data would often not refer to the devices except to check that they had reached a given location.

Furthermore, other people played a part in the game whether as a co-player, passer-by or in-game character. However, the strongest sense of presence and most favourable feedback was reserved for the co-operative aspect of the game with the co-player. Furthermore data 
from the questionnaires and interviews pointed further to the benefit of a co-operative game design, for example the feature was frequently mentioned as a favourite. However, the data also points to the need to provide a better experience for the second player as comments suggested that at times this could be a little boring; thus, there is a need to more carefully design the feelings for all players. From a presence perspective, it is was unsurprising that such an approach would lead to the co-player being seen as the most present, especially when compared to the computergenerated elves and the fact that non-players were not integrated into the game.

One of the main challenges with such games is to provide an experience that is continuous; by this we mean that players constantly feel present in the experience at different points in the game. However, data from this study points to players experiencing changing feelings of presence throughout the gaming experience. These extend from feeling out of the game experience while navigating between game content locations, through to feeling the lowest sense of presence at the very start of the game and conversely feeling change in presence when leaving the game. The problems at the start of the game can in part be explained by the fact that the players are becoming familiar with the technology and the gaming environment. Indeed, previous work using an earlier version of the system via augmented reality visors pointed to the need for an appropriate training scenario to overcome this problem. However, even the training scenario within this version did not provide a strong enough experience so as to overcome their low sense of presence at the start. This in turn points to the need for such training scenarios to be better integrated with the game content so as to encourage a sense of presence.

The study also points to players feeling as if they are dropping out of the game experience when moving between locations. This was manifested initially in the observation data which pointed to decreasing interest in the game world (e.g. checking devices less frequently) through to people indicating that they were navigating by real world rather than game elements between locations. While it is not possible from the data to draw any specific design conclusions, a number of possible areas for exploration are considered relevant, for example integrating real people and objects into the game environment, providing more content and possibility for action between locations or making greater use of real street ambience such that people feel excited or scared when walking through certain areas. However, such an approach would require an understanding of how sense of place can be utilized and altered within such a context.

The time portals were the highest rated aspect of the game, although they are unique to this particular game there a number of properties which are applicable across other experiences. For example the time portals are a very physical and collaborative experience for both players, often requiring them to run, crouch and agree how to enter them. Furthermore, players must enter them within a set period of time. Therefore, adding aspects to the game play which encourage such behaviour is desirable. While the Likert questions did not specifically ask players on their attitudes towards the time portals the responses during the interviews, written responses and body language would suggest that the players did respond as if they actually there - even if they seemed patently out of place with respect to the overall city. This would imply that unlike some of the comments received about buildings etc. being out of place with respect to time periods, when an experience is clearly not-real (e.g. time travel) people suspend their disbelief and engage more fully in the game. Conversely, when there is a clear link to reality, they are slightly more critical. This view was also reflected in the opinions of the future time period. Therefore, there seems to be a contextual issue which requires further investigation for example when should there be a clear link between the real and virtual elements, and when should there be a clear break.

Data from the questionnaires and interviews pointed to the need to carefully design challenges such that they are not too easy to solve and match the players' abilities. For example players often commented that a task was too easy to complete, and thus was not challenging enough. However, given the novelty factor of such technologies coupled with the occasional technical problems care should be taken to ensure that emphasis is on completing the challenge and not overcoming usability aspects.

Audio elements were deemed to be important within the game play, with many noting that they provided a stronger sense of presence than the graphical elements. The sounds provided navigational cues as well as content and encouraged collaboration between players and decision making. Although the data from this part of the study is limited, it does point to the need to more fully consider the soundscapes, in particular as a way of encouraging collaboration or in order to replace graphical elements which are difficult to see or are problematic.

\section{Conclusions}

This paper presented a study of an augmented reality location-based game which aims to alter the player's sense of presence as they undertake a range of tasks. As noted in the background literature section, it is the nature and availability of tasks and actions which shapes the players sense of presence, this position was validated within the study. This extends from when players have no clear tasks 
and hence available actions between content locations, to the nature of the tasks they are completing. For example tasks which are too easy are viewed negatively; an issue which has already been highlighted with respect to game flow. However, tasks which are short yet comparatively easy to complete but contain strong physical elements such as the time portals are generally well received. Also when an environment is perceived as not providing any available actions, as was often the case between locations, players appeared to leave the game world and thus experience a drop in presence.

Measuring presence within urban environments is a difficult task, not least as traditional laboratory-based studies are not appropriate. The methods presented in this paper provide an approach that allows for the identification of common findings across different data sources. On their own questionnaires only provide an overall view of the gaming experience, however, when used in combination with data from direct observation they provide a useful method of structuring interviews. Furthermore, video analysis provides another method of exploring user behaviour in particular clear and obvious movements, which in turn when explored in connection with other data sources can help shed light on the actions and feelings of the users.

\section{References}

1. Bailenson JN, Blascovich J, Beall AC, Loomis JM (2001) Equilibrium revisited: mutual gaze and personal space in virtual environments. Presence Teleoperators Virtual Environ 10:583598

2. Ballagas RA, Kratz SG, Borchers J, Yu E, Walz SP, Fuhr CO, Hovestadt L, Tann M (2007) REXplorer: a mobile, pervasive spell-casting game for tourists. Conference on human factors in computing systems, San Jose, CA, USA, pp 1929-1934

3. Benford S, Crabtree A, Reeves S, Flintham M, Drozd A, Sheridan J, Dix A (2006) The frame of the game: blurring the boundary between fiction and reality in mobile experiences. Proceedings the 2006 ACM CHI conference on human factors in computing systems. ACM, Montreal, pp 427-436

4. Benyon D, Smyth M, O'Neill S, McCall R, Carrol F (2006) The place probe: exploring a sense of place in real and virtual environments. J Presence Tele Oper Virtual Environ 15(6):668687
5. Cheok A, Goh KH, Liu W, Farbiz F, Fong SW, Teo SL, Li Y, Yang X (2004) Human Pacman: a mobile, wide-area entertainment system based on physical, social, and ubiquitous computing. Personal Ubiquitous Comput 8(2):71-81

6. Csikszentmihalyi M (1990) Flow: the psychology of optimal experience. Harper Perennial, New York

7. Ericsson M (2003) Enchanting reality: a vision of big experiences on small platforms. In: Copier M, Raessens J (eds) Level up. Digital games research conference 4-6 November 2003 proceedings. Universiteit Utrecht, Utrecht

8. Gibson JJ (1979) The ecological approach to visual perception. Erlbaum, Hilldale

9. Gustavson P (2001) Meanings of place: everyday experience and theoretical conceptualizations. J Environ Psychol 21:5-16

10. Herbst I, Braun A, McCall R, Broll W (2008) TimeWarp: interactive time travel with a mobile mixed reality game. In: Proceedings of the 10th international conference on human computer interaction with mobile devices and services (Amsterdam, The Netherlands, 2-5 September 2008). MobileHCI '08. ACM, New York, NY, pp 235-244

11. Huizenga J, Admiraal W, Akkerman S, Dam GT (2007) Learning history by playing a mobile city game. In: Remenyi D (ed) Proceedings of the 1st European conference on game-based learning (ECGBL) October 2007, University of Paisley, Paisley, Scotland. Academic Conferences Limited, Reading, pp 127-134

12. Lindt I, Ohlenburg J, Pankoke-Babatz U, Ghellal S (2007) A report on the crossmedia game epidemic menace. ACM Computers in Entertainment (CIE) 5(1)

13. Mantovani G, Riva G (1999) "Real" presence: how different ontologies generate different criteria for presence, telepresence, and virtual presence. Presence Teleoper Virtual Environ 5(8):538-548

14. Milgram P, Kishino F (1994) A taxonomy of mixed reality visual displays. IEICE Trans Inf Syst 77:1321-1329

15. Montola M (2005) Exploring the edge of the magic circle. Defining pervasive games. DAC 2005 conference, December 3. IT University of Copenhagen

16. Piekarski W, Thomas B (2002) ARQuake: the outdoor augmented reality gaming system. Commun ACM 45(1):36-38

17. Relph E (1976) Place and placelessness. Pion Books, London

18. Sweetser P, Wyeth P (2005) Gameflow: a model for evaluating player enjoyment in games. ACM Comput Entertain 3(3):1-24

19. Vorderer P, Wirth W, Gouveia FR, Biocca F, Saari T, Jäncke F, Böcking S, Schramm H, Gysbers A, Hartmann T, Klimmt C, Laarni J, Ravaja N, Sacau A, Baumgartner T, Jäncke P (2004) MEC spatial presence questionnaire (MEC-SPQ): short documentation and instructions for application. Report to the European community, project presence: MEC (IST-2001-37661). Online. Available from http://www.ijk.hmt-hannover.de/Presence

20. Wetzel R, Lindt I, Waern A, Johnson S (2008) The magic lens box: simplifying the development of mixed reality games. In: Proceedings of the 3rd international conference on digital interactive media in entertainment and arts (Athens, Greece, 10-12 September 2008). DIMEA '08, vol. 349. ACM, New York, NY, pp 479-486 